\title{
The Research on Quantitative Identification of Upper Limb Motion Based on SEMG
}

\author{
Changsong Li and Yiqi Zhou*
}

School of Mechanical Engineering, Shandong University, Jinan, P.R. China

\begin{abstract}
Surface electromyography signal (sEMG) can reflect nerves and muscles' motion to a certain degree and has great practical value in clinical medical as well as in the medical rehabilitation field, such as nerves and muscles disease diagnosis, muscles function evaluation and artificial limb control, which achieve certain development and is applied in the joint motion information identification. Based on the characteristics of hemiplegia patients whose one side limbs motion function is destroyed, this paper researches on the quantitative identification of upper limbs motion based on sEMG to understand the patients' motion intention and consequently supply automatic motion control to the patients. Meanwhile, this paper focuses on feature extraction and quantitative identification of sEMG as a key technology. The MLPs based on the Bayesian Regulation was used to identify the degree of the elbow joint of the subject, and reduce the disadvantage of the normal MLPs which is lack of the enough ability of extension towards this complicated Sub-Gaussian random signals. The research will be helpful to develop the EMG signals into the actual application of the medical robot.
\end{abstract}

Keywords: SEMG, feature extraction, quantitative identification.

\section{INTRODUCTION}

Upper limb rehabilitation robot system is the robot technology which was in the field of clinical medicine and produce a complementary or alternative doctor completed the limb rehabilitation training of special robot system, its appearance has opened up a new technical way for upper limb hemiplegic rehabilitation, and makes up for the clinical treatment of the movement of the problems mentioned above. Upper limb rehabilitation robots break the limitation of doctor-patient between one to one, will not only the physician from heavy, repeated training mission to make it more focus on the treatment improvement, and making it possible to focus on medical and remote rehabilitation medical; In addition, the rehabilitation robot through intelligent human-machine interface, can provide different strength in different period of rehabilitation patients, different modes of training, active exercise consciousness motivate patients to enhance confidence, enhance the rehabilitation confidence of patients; Data of sensor to measure the kinematics of human body, physiological utilization of rehabilitation robots provide, provide an objective basis for improving and optimizing the rehabilitation physician [1]. Therefore, the research, the development of upper limb rehabilitation robot system will promote the medical and engineering resources complementary, is important practical significance to the development of the cross subject and social progress of rehabilitation.

Surface electromyography signal (sEMG) is from the skin surface through the guidance of the electrode, amplification, display and record down the biological electrical signal when neuromuscular system activity, the signal form has great randomness and uncertainty. Because of sEMG exists different degree of correlation with muscle function and activity states, which can reflect the neuromuscular activity to some extent, at the same time sEMG has the advantages of non-invasive, real-time and multiple targets, neuromuscular disease diagnosis in clinical medicine, rehabilitation medicine in the field of muscle function evaluation, as well as the fatigue assessment in sports science, exercise technology and reasonable analysis and so on all have important practical value [2].

There is a lot of researches focus on the pattern recognition of sEMG, so as to distinguish different limb motion mode, realize the control of multi degree of freedom prosthetic. However, in a single movement mode, the motion state of the prosthesis such as movement speed, movement range and the position of the arm can't control. This is mainly due to the complexity of the electromyographic signal producing mechanism and the complex relationship between the electromyographic signal and human self motion control system, makes the relationship between sEMG and arm motion state is difficult to determine. The process of pattern recognition using sEMG can be expressed as the electromyographic signal collection, signal preprocessing (optimization), feature extraction and pattern recognition. First by analyzing the electromyographic signal to extract the characteristic value, and then provided input pattern recognizer for identification. In order to accurately realize the gesture recognition, feature extraction and pattern recognition are the key to the whole system [3].

Due to the original action sEMG has a typical non-linear and non-stationary characteristics, so from the perspective of stationary random signals (such as statistical characteristic analysis and Fourier transform) to obtain the recognition rate 
has big error, and from the angle of non-stationary (timefrequency analysis) or nonlinear angle (nonlinear feature extraction) or a combination of both angle (combining empirical mode decomposition and nonlinear characteristics) to analyze signal can obtain better recognition rate.

In order to extract the corresponding feature effectively, should be the first in-depth analysis of surface electromyography signal, to master certain prior knowledge. Then according to the action surface electromyographic signal choose the appropriate features and treatment methods, and then input the corresponding classifier for identification. The effect of pattern recognition is the most effective tool to test whether the appropriate feature extraction. The system principle diagram of Robot based on sEMG was shown in Fig. (1).

\section{SURFACE EMG SIGNAL PROCESSING}

Collected for processing the original action surface EMG signal including data collection, filtering, model selection, feature extraction and pattern recognition, as shown in Fig. (2).

\subsection{Feature Extraction}

Different feature extraction method is based on the original surface EMG action seen as different models, then the corresponding feature extraction or feature space conversion, and then calculate the corresponding typical characteristics, for identification [4]. When performing model selection, a priori knowledge, that mastery of the original action surface EMG is essential. Select the appropriate model determines whether the extracted features is appropriate, also decided to identify the good and bad effects.

In the methods described herein taken in, statistical feature extraction will be seen as the original action surface EMG same statistical characteristics of stationary random signal, the Fourier transform is built on the basis of stationary random signal, time-frequency analysis method sucked original action seen as non-stationary surface EMG signals, nonlinear feature extraction sucked original action deemed non-linear surface EMG signals, to calculate the typical nonlinear characteristics and identified. Empirical mode decomposition method is also from the perspective of non-stationary nonlinear original action surface EMG decomposition and feature extraction. Percentage of energy is from the perspective of the energy distribution of the original action surface EMG signals were identified. Because the original surface EMG action with a typical non-linear non-stationary characteristics [5], therefore from the perspective of a stationary random signals (such as statistical feature analysis and Fourier transform) the recognition rate obtained large error, and

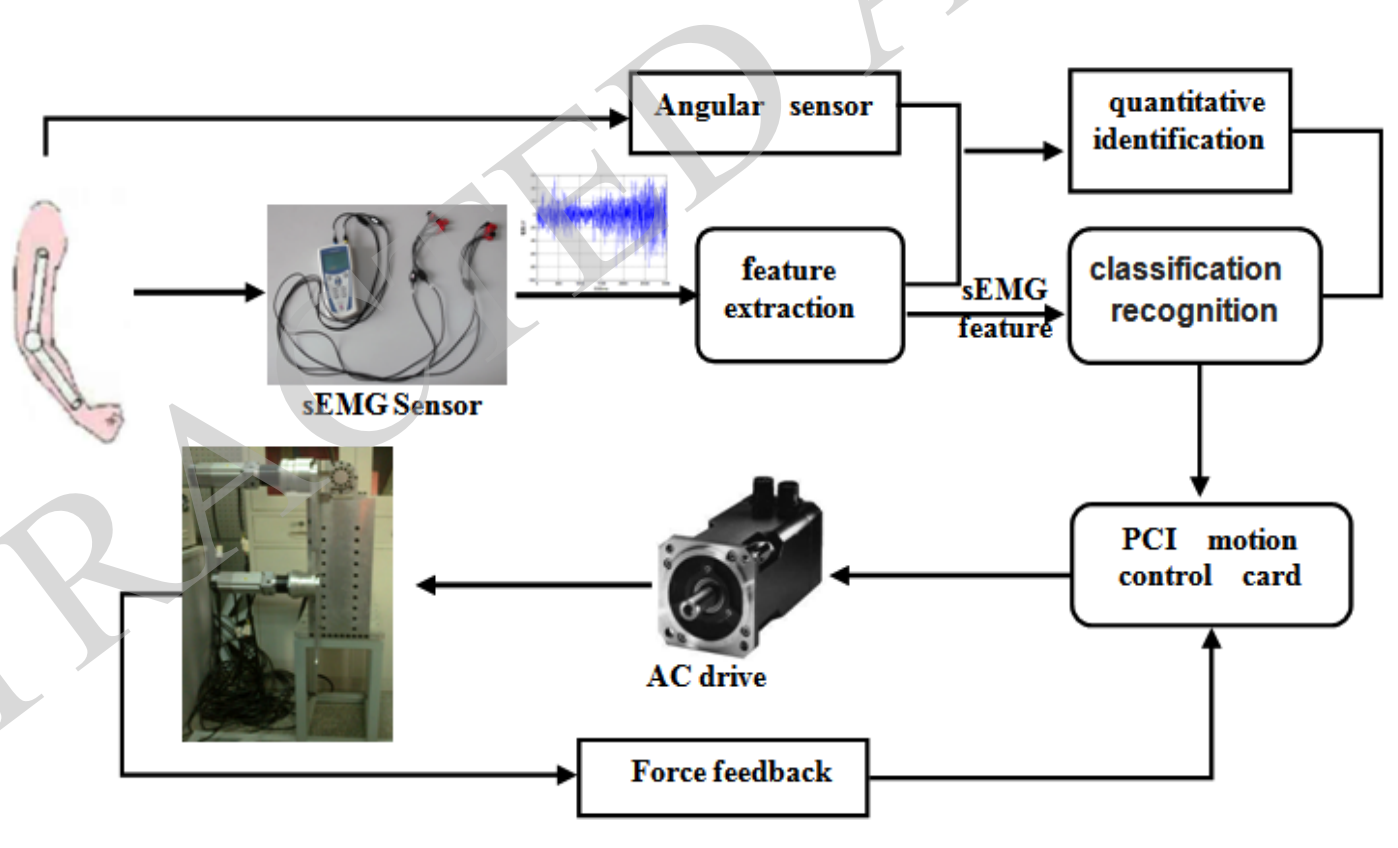

Fig. (1). The system principle diagram of Robot based on sEMG.

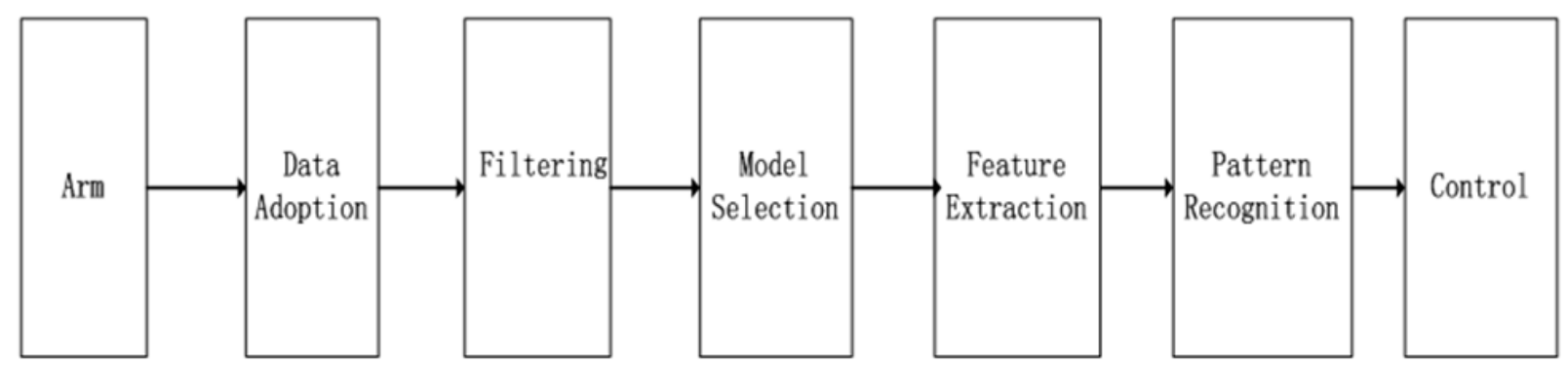

Fig. (2). The flow chart of signal processing. 
from the perspective of non-stationary (time-frequency analysis) or nonlinear angle (non-linear feature extraction), or a combination of both the departure angle (empirical mode decomposition and nonlinear characteristics of the combination) of the signal can be analyzed to obtain the ideal identification rate. The frequently used methods of feature extraction were shown in Fig. (3). Feature extraction method using Combined with feature recognition was shown in Fig. (4).

(1) Arithmetic Mean Value, which as the general description of the centure of the data probability distribution.

$\bar{x}=\frac{1}{N} \sum_{i=0}^{N-1} x(i)$

(2) Mean absolute difference in time-domain analysis.

$x=\frac{1}{N} \sum_{i=0}^{N-1}|x(i)|$

(3) Range is the difference between the Max and the Min of the sample.

$$
x=x_{\max }-x_{\min }
$$

(4) The standard deviation reflect concentration and dispersion degree of the random variable.

$$
\begin{gathered}
x=\frac{1}{N} \sum_{i=0}^{N-1} x(i) \\
\delta=\sqrt{\frac{1}{N} \sum_{i=0}^{N-1}[x(i)-x]^{2}}
\end{gathered}
$$

\subsection{Pattern Recognition}

Back propagation network is a multilayer of nonlinear differential function weight training feed forward neural networks. The meaning of error of back propagation is: the network output error at the little direction was adjusted according to the connection weights of output error to each layer of neurons in the network of reverse, and finally achieve the desired output. BPNN has the characteristics: (1) to realize the nonlinear mapping of different dimension

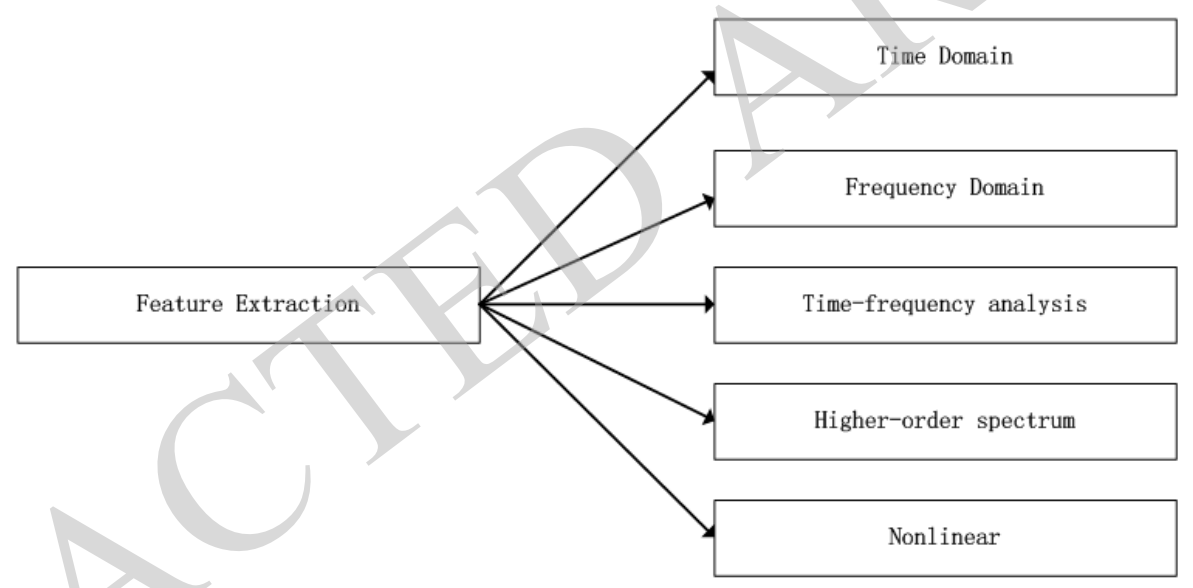

Fig. (3). The frequently used methods of feature extraction.

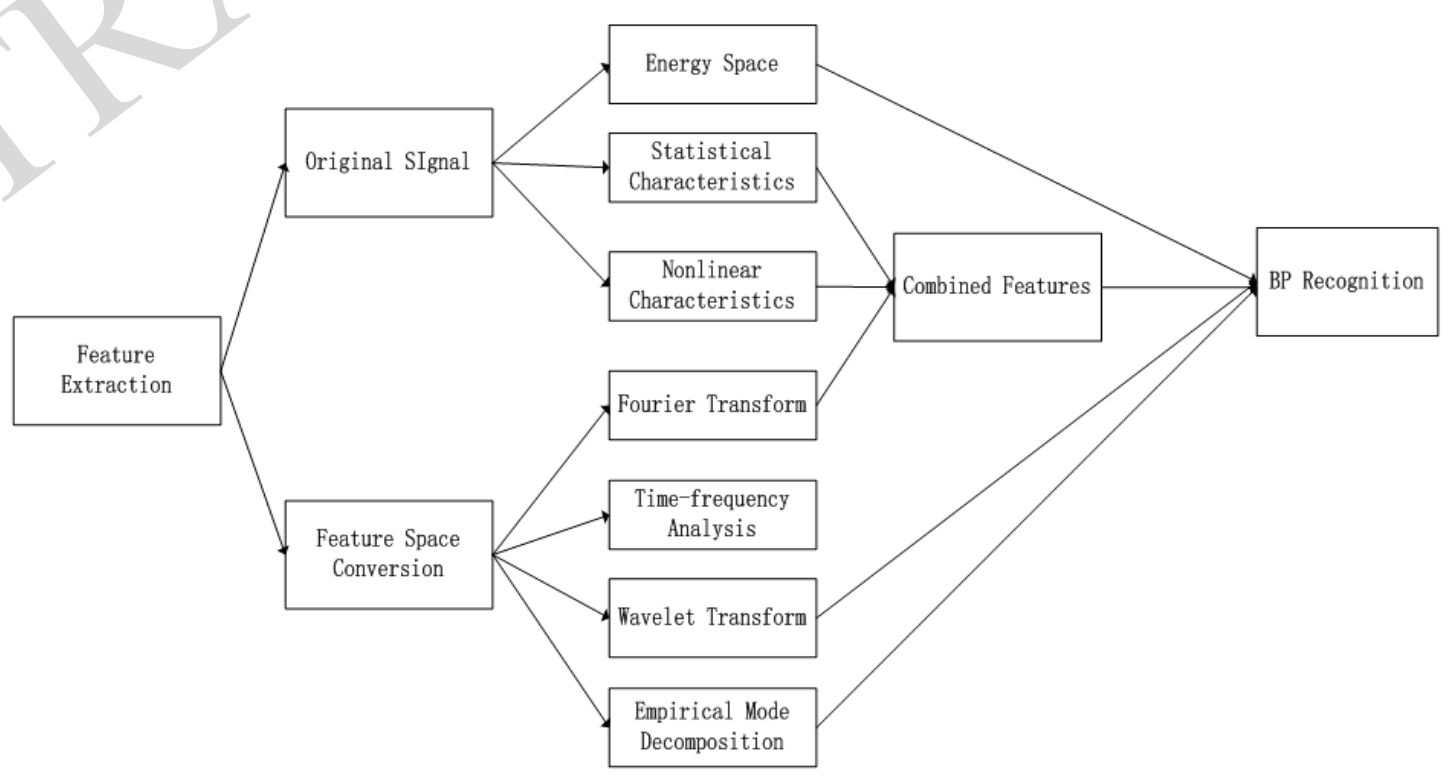

Fig. (4). Feature extraction methods using Combined with feature recognition. 
space; (2) according to the samples self-learning; (3) distributed knowledge expression; (4) parallel information processing (5) there is a certain generalization capability to unknown information. According to the research, BPNN can be used as a standard type of nonlinear approximation: with regard to any function of the real space, as long as it satisfies certain conditions, there must be only with a single hidden layer BPNN as the best for its optimal error back propagation learning algorithm which consists of two steps: the first is the progress of forward input [6], the second is the process of output error back propagation. The so-called positive communication refers to the input signal applied to the input layer of the network layer to the output layer transfer which get the output response. While the output error back propagation is the output error value by the output to the input layer began to reverse the spread of, each layer of the network weight changes are based on spread to the error of the layer to decide. At the start of training before all weights must be initialized. The choice of initial value is a important problem. In the absence of prior knowledge of what a good contrast, usually by initializing the random number to small. In the view of the characteristics of BP network, this paper uses the BP neural network for motion pattern classification of EMG signals processed, to identify the joint flexion extension motion do the motion state of the joint trajectory prediction. The BP network consists of three layers, [7] the first layer is the input layer, there are four nodes, the input feature 4 road sEMG values; the last layer is the output layer, a node, output angle signal. Full interconnection mode is adopted between the different layers of neurons, between the same layer neurons does not exist mutual connection; In the network, the input layer and the hidden layer using bipolar $S$ shape function. The neural network structure as shown in Fig. (5).

It is needed to pay attention to the middle layer which is to determine the number of hidden units. There is obvious influence to the performance in BP network nodes on the network. The hidden layer node is too small, the network may not be able to solve the problem of: too much, and the hidden layer [8], the network may be training samples to accurately describe and not to the new input pattern precise description. There are 3 formulas can be used for reference:

$$
\sum_{i=0}^{n} C_{n 1}^{i}>k
$$

k----sample number

n1----- The number of hidden layer units

n-----The number of input units

First training on BPNN, from the application environment and pick out a few sample data and the corresponding class labels weights and linear bias of BPNN adjustment, until we get the relationship between input and output right [9], by the end of that network convergence. The network after training, for each new test samples coming discriminant results directly by the trained BPNN by forward calculation can get the sample category. Training and simulation using MATLAB7.1 for neural network. The establishment of BP network, prediction and angle signal. Block diagram as shown in Fig. (6).

\section{CONCLUSION}

The research is the acquisition and feature extraction based on sEMG, and joint angle signal synchronous acquisition, analysis, quantitative information on the joint motion recognition, establish a quantitative relationship between the model and the joint angle sEMG signal; On this basis, quantitative relationship model will be collected sEMG input has been built, the output prediction joint angle, thereby identify movement intentions, exercise planning, driving the robot to drive upper limb rehabilitation in accordance with the patient's movement intent.

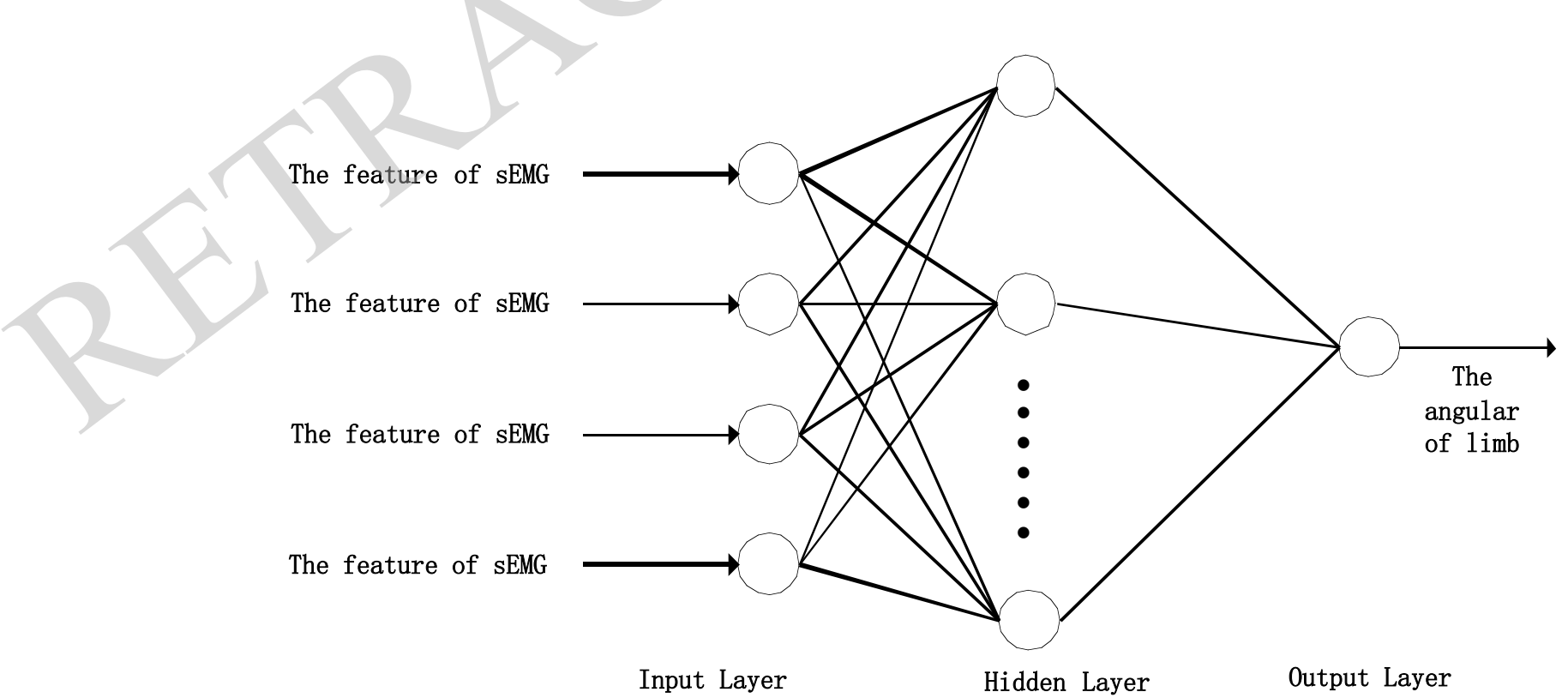

Fig. (5). The structure of BP neural network. 


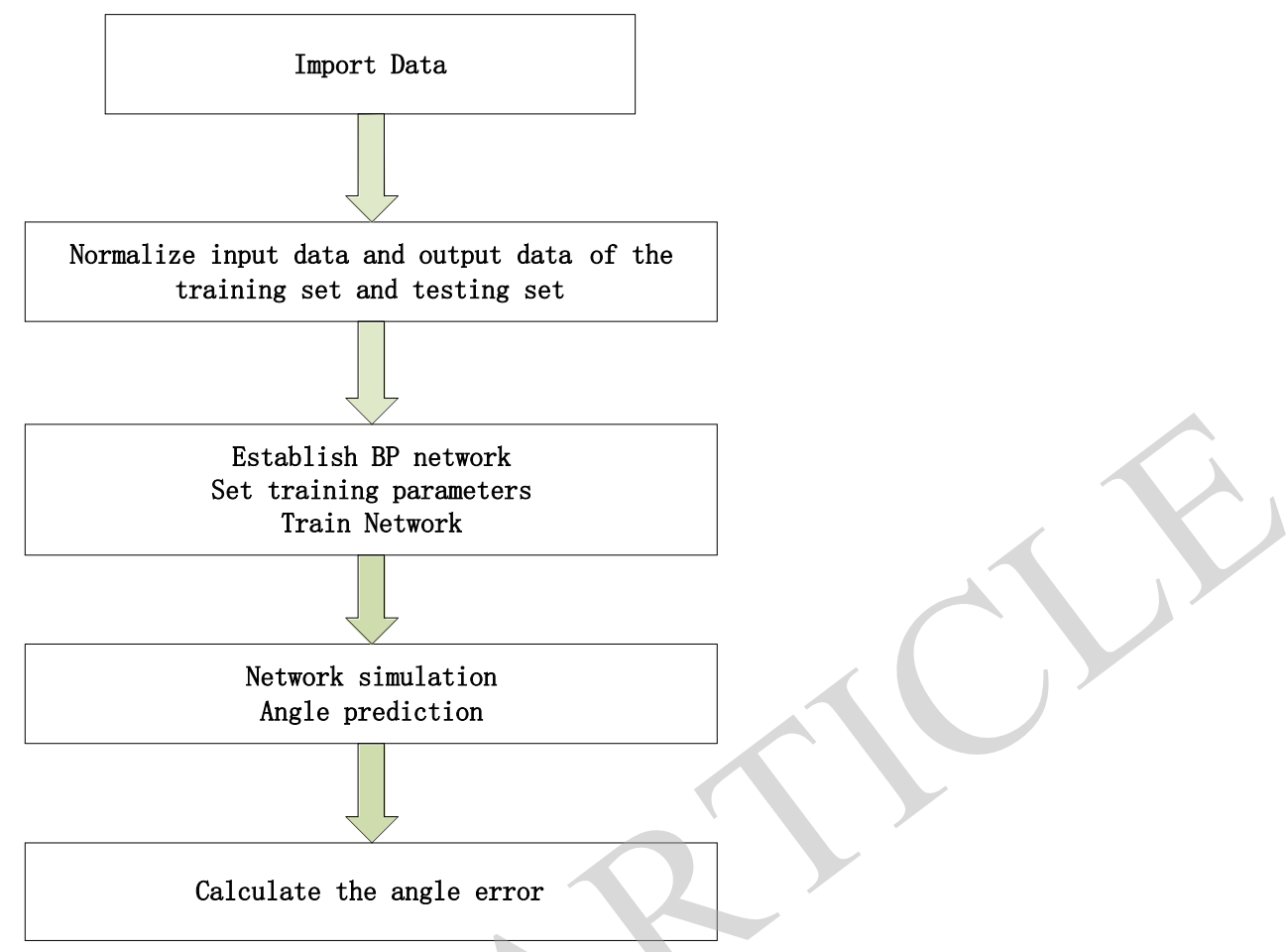

Fig. (6). Quantitative Identification of the block diagram.

\section{CONFLICT OF INTEREST}

The authors confirm that this article content has no conflicts of interest.

\section{ACKNOWLEDGEMENTS}

The research was supplied by the Key Laboratory of High-efficiency and Clean Mechanical Manufacture at Shandong University, Ministry of Education, Jinan, China. At the same time, it was also supplied by the Key Laboratory of computer application at Shandong Women University. The corresponding author of the paper are Zhou Yiqi (School of Mechanical Engineering, Shandong University) and Li Ying (School of Information and Technology, Shandong Women University).

\section{REFERENCES}

[1] C.G. Burgar, P.S. Lum, and P.C. Shor, "Development of robots for rehabilitation therapy: the Palo Alto VA/Stanford xperience," Journal of rehabilitation research and development, vol.37, no.6, pp.663-73. 2000.
[2] P. Duhamel, "Implement of "Sp lit2Radix" FFT Algorithm s for Complex, Real, and Realsymmetric Data," IEEE Trans. Acoust Speech. Signal Processing, 1986.

[3] E. Lamounier, A. Soares, and A. Andrade, "A virtual prosthesis control based on neural networks for EMG pattern classification," In: Proceeding of the Artificial Intelligence and Soft Computing, 2002.

[4] J.D. Zhao, Z.W. Xie, and L. Jiang, "Levenberg Marquardt based neural network control for a five-fingered prosthetic hand," In: Proceeding of the 2005 IEEE International Conference on Robotics and Automation, pp.4482-4487, 2005.

[5] L. Guo, and L. Ji, "Relation between patients' active force and effect of robotic therapy for rehabilitation," In: Proceeding of the 27th IEEE international Conference on Engineering in Medicine and Biology Society, vol. 5, pp. 5044-5046, 2005.

[6] M. Lei, Z. Wang and Z. Feng, "Detecting nonlinearity of action surface EMG signal," Physics Letters, vol. 290, no.5-6, pp.297303, 2001.

[7] I. Mrazova, "The robustness of BP-networks," Artificial Neural Networks, 1995

[8] T. Nef, and R. Riener, "ARM in-design of a novel arm rehabilitation robot," In: Proceeding of the 9th IEEE Conference on Rehabilitation Robotics, pp. 57-60, 2005.

[9] X. Q. Wang, Y. H. Wang, and S. H. Qian, "New technology for fault diagnosis based on wavelet denoising and modifies exponential time-frequency distribution," Mechanical Engineering, vol. 14, no. 3,2001 .

(C) Li and Zhou; Licensee Bentham Open.

This is an open access article licensed under the terms of the (https:/creativecommons.org/licenses/by/4.0/legalcode), which permits unrestricted, noncommercial use, distribution and reproduction in any medium, provided the work is properly cited. 